\title{
Educación vial y movilidad en la Infancia
}

\author{
Fernando Martín Poó \\ Universidad Nacional de Mar del Plata - Mar del Plata - Argentina \\ Soledad Susana López \\ Universidad Nacional de Mar del Plata - Mar del Plata - Argentina \\ Jeremías Tosi \\ Universidad Nacional de Mar del Plata - Mar del Plata - Argentina \\ María Isabel Nucciarone \\ Universidad Nacional de Mar del Plata - Mar del Plata - Argentina \\ Rubén Daniel Ledesma \\ Universidad Nacional de Mar del Plata - Mar del Plata - Argentina
}

\begin{abstract}
Resumen
La motorización creciente de los desplazamientos ha convertido al ambiente urbano en un lugar cada vez más hostil para los niños y niñas, quienes se encuentran entre los grupos humanos más vulnerables del tránsito. En esta etapa del desarrollo, la educación puede ser una herramienta clave para prevenir comportamientos de riesgo y promocionar hábitos de movilidad más saludables. El presente trabajo discute algunos aspectos relacionados con la educación vial en la infancia. Se destaca la importancia de considerar los patrones de movilidad típicos de cada edad, y de promover el uso de medios de transporte más saludables, equitativos y sustentables. Se señala además la necesidad de complementar las acciones educativas con intervenciones multi-sectoriales a diferente nivel.
\end{abstract}

Palabras clave: Educación; infancia; tráfico.

\section{Educação no trânsito e mobilidade na infância}

\section{Resumo}

A crescente motorização e os deslocamentos têm transformado o meio ambiente urbano num lugar cada vez mais hostil para as crianças, que se encontram entre os usuários mais vulneráveis do trânsito. Nessa etapa do desenvolvimento, a educação pode ser uma ferramenta chave para prevenir o comportamento de risco e promover hábitos de mobilidade mais saudáveis. O presente trabalho discute alguns aspectos relacionados à educação infantil no trânsito (nas ruas). Destaca-se a importância de considerar os padrões de mobilidade típicos de cada idade e de promover o uso dos meios de transportes mais saudáveis, equitativos e sustentáveis. Além disso, assinala-se a necessidade na complementação das ações educativas com intervenções multi-setoriais em diferentes níveis.

Palavras-Chave: Educação; infância; trânsito.

\section{Childhood education and mobility}

\begin{abstract}
The increasing motorization of mobility has turned urban environment in a complex and dangerous place for children. As a consequence they are one of the most vulnerable groups of traffic users. In this stage of development, education could be a key tool for preventing risky behaviour and promoting more healthy mobility habits. The present study discusses some aspects related to children traffic education. The necessity of taking into account the patterns of mobility in different developmental stages is highlighted. Also the relevance of promote the use of health, sustainable and equitable means of transport. The complexity of the problem requires that educational interventions are complemented with multisectoral actions at different levels.
\end{abstract}

Key words: Education; childhood; traffic. 


\section{Introducción}

Durante la última década Argentina ha experimentado un incremento notable del parque automotor, sobre todo de vehículos personales. La motorización progresiva de los desplazamientos ha convertido al tránsito en un ambiente más hostil, especialmente para los grupos de usuarios más vulnerables, como los niños y las niñas. Entre las consecuencias negativas de esta situación se destacan las lesiones y las muertes producidas por siniestros, pero también otras como el deterioro en la calidad del ambiente urbano. Se trata de un problema complejo, por lo cual los cambios a mediano y largo plazo dependerán tanto de las políticas de Estado como del compromiso de las comunidades en su conjunto. En este contexto, la educación vial, definida como la transmisión de conocimientos y la adquisición de habilidades (Groeger, 2011) para un uso seguro, responsable y sustentable del transporte resulta una tarea clave.

La educación vial debería iniciarse en los primeros momentos de la infancia, con la participación activa de las familias y las instituciones educativas, pero también de otros sectores, como la salud y el transporte. Más allá del ámbito en que se desarrolle, resulta fundamental reflexionar críticamente sobre los objetivos, contenidos y enfoques que guían actualmente la tarea educativa. Para ello, es imprescindible considerar las nuevas demandas y necesidades en materia de movilidad, transporte y tránsito. En este trabajo se discuten varios aspectos referidos a la educación vial infantil. Se parte de suponer la necesidad de un cambio de enfoque que permita: (a) favorecer comportamientos seguros focalizados específicamente en los modos de movilidad propios de cada etapa evolutiva, y (b) promover activamente actitudes y conductas que contribuyan al desarrollo de un modelo de transporte más saludable, equitativo y sustentable.

\section{Infancia y Seguridad Vial}

Los incidentes de tránsito conllevan una alta tasa de mortalidad y lesiones en todo el mundo (World Health Organization [WHO], 2013). La Organización Mundial de la Salud estimó que 1,24 millones de personas pierden la vida anualmente como consecuencia de siniestros viales, lo que posiciona a estos eventos como la novena causa de muerte en todo el planeta para todos los grupos de edad. Además, la mayoría de las muertes involucran a los usuarios más vulnerables del tránsito entre los que se incluyen peatones, ciclistas, motociclistas y pasajeros de transporte público. Por otra parte, los incidentes viales no sólo provocan decesos, sino que también producen entre veinte y cincuenta millones de lesionados por año, muchos de las cuales sufren discapacidades de distinta gravedad. Al sufrimiento personal que estos hechos generan deben sumarse los costos materiales a nivel individual y social ya que los siniestros viales producen una sobrecarga evitable sobre los servicios de emergencias y altos costos económicos para todo el sistema de salud.

Si se consideran los datos estadísticos referidos a la infancia, el problema adquiere más relevancia. Según la Organización Mundial de la Salud [OMS] (2009) durante el año 2004 los traumatismos sufridos en el contexto vial representaron la segunda causa de mortalidad para los niños entre 5 y 14 años de edad, y la principal causa de muerte para los que tienen entre 10 y 19 años. A nivel mundial, la infancia representa el $21 \%$ de los siniestros de tránsito con consecuencias fatales. Este porcentaje es aún más elevado en países de ingresos medios y bajos (30-40\%). Los datos indican que durante la infancia el riesgo de sufrir heridas fatales o no fatales como peatón es mayor que a cualquier otra edad. Por otra parte, la probabilidad de que un niño o una niña sufra un incidente vial, aumenta cuando participa en el tránsito como ciclista o acompañante de vehículos motorizados (National Safe Kids Campaign, 2004; Romano, \& Kelley-Baker, 2015). Las previsiones para el futuro no son optimistas ya que se estima que para el año 2015 los incidentes de tránsito serán la fuente principal de discapacidad y de muerte en niños y niñas mayores de 5 años (OMS, 2009).

En Argentina, los incidentes viales también representan un serio problema de salud pública. Según Fleitas (2010), entre 1997 y 2008 murieron 4.107 niños menores de 14 años por incidentes de tránsito, de los cuales 1.286 fueron niños de 4 años o menores. Según la Agencia Nacional de Seguridad Vial los niños de 5 a 14 años representan un $5,2 \%$ del total de víctimas fatales por incidentes de tránsito, y un $5,6 \%$ del total de víctimas no fatales (Agencia Nacional de Seguridad Vial, 2011).

La situación es aún más preocupante si se considera que se trata, en gran medida, de un problema que podría prevenirse mediante programas de intervención pertinentes. En los últimos años, la Argentina ha tomado medidas estratégicas con el objetivo de introducir mejoras en la seguridad vial. Entre estas medidas se encuentra la conformación de la Agencia Nacional de Seguridad Vial (ANSV) que coordina e implementa el Plan Nacional de Seguridad Vial. El plan prioriza tres ejes (a) concientización, (b) prevención y (c) control. Se destaca también el Programa de Fortalecimiento de la Educación Vial elaborado en colaboración entre el Ministerio del Interior y el Ministerio de Educación, cuya finalidad es incorporar la educación vial como contenido curricular para las instituciones educativas del país. Este programa enfatiza la necesidad de sustituir un enfoque centrado en las "vías", "normas" o "caminos", por una mirada focalizada en el usuario del tránsito, independientemente del medio de transporte que utilice. Promueve una perspectiva que se denomina Educación del transeúnte, y aborda al mismo tiempo ejes tales como la educación para la salud, la educación para la convivencia y la ciudadanía, y la educación ambiental. El programe tiene como objetivo lograr el desarrollo de una moral autónoma y solidaria, que promueva la conformidad con las normas para resguardar el bienestar propio y ajeno, y no como mera obediencia a la autoridad. 
Iniciativas como estas resultan muy importantes para abordar el problema de los comportamientos de riesgo en el ambiente vial. Sin embargo, existen otros aspectos del tránsito y la movilidad que también afectan la salud pero que no han recibido suficiente atención hasta el momento. Concretamente, el incremento del parque automotor afecta considerablemente la calidad de los ambientes urbanos (Wolf, \& Gillham, 1991). En la Argentina, a principios del año 2012 se estimaba que había un automóvil cada cuatro personas. Esto equivale a casi 10 millones de unidades en circulación. Esta proporción, lejos de disminuir, parece que continuará aumentando con los años. Algunas de las consecuencias del aumento continuo del número de vehículos motorizados son: la creciente polución ambiental, la contaminación sonora y la reducción del espacio para formas de movilidad alternativas (Wolf, \& Gillham, 1991).

\section{Infancia y Movilidad}

Es evidente que los niños poseen características específicas (a nivel cognitivo, emocional, conductual y físico) así como formas de movilidad que los convierten en un grupo vulnerable en el contexto vial (Oxley, Congiu, Whelan, D'Elio, \& Charlton, 2008; OMS, 2008; Zeedick, Wallace, \& Spry, 2002). El tránsito es un ambiente complejo, cambiante y exigente, en el cual se ponen en marcha una diversidad de procesos perceptivos, atencionales y de toma de decisiones que en ellos se encuentran en desarrollo. Por lo tanto, resulta fundamental orientar y ajustar la educación vial a sus necesidades, capacidades y funciones en el tránsito.

Las diferentes etapas del desarrollo se caracterizan por cambios en los niveles de independencia y movilidad y la posibilidad de elegir diferentes modalidades de transporte. A medida que se producen esos cambios aparecen nuevas fuentes de peligro. Al respecto, datos epidemiológicos muestran que el patrón de lesiones durante la infancia se modifica con el paso del tiempo (Waisman, Nuñez, \& Sanchez, 2002). Durante la primera infancia predominan las lesiones ocurridas en el hogar, mientras que las lesiones producidas en el contexto vial aumentan en la medida en que crece la autonomía en los desplazamientos urbanos. El patrón de las lesiones de tránsito también se modifica con el crecimiento. Durkin, Laraque, Lubman y Barlow (1999) observaron que en la primera infancia son más comunes las lesiones como peatones o pasajeros de autos mientras que las lesiones como ciclistas aumentan entre los 9 y los 14 años.

Cuando los niños y niñas se desplazan en el contexto vial, se enfrentan a un ambiente cuyo diseño, en general, no contempla sus características ni sus necesidades específicas. Además, muchas situaciones del tránsito implican evaluaciones y decisiones complejas, para las cuales no siempre están preparados. Por ejemplo, Oxley y cols. (2008) han puesto de manifiesto la dificultad involucrada en la "decisión de cruzar una calle", la cual implica un amplio rango de habilidades perceptuales, atencionales y de planificación. Cruzar la calle supone, entre otras tareas, realizar estima- ciones sobre la distancia y velocidad de un vehículo que se aproxima. Mientras que para los adultos esta estimación se realiza automáticamente, los niños más pequeños consideran que la variable relevante es la distancia respecto del vehículo, independientemente de la velocidad a la que circula. Esto puede derivar en decisiones erróneas al momento de cruzar sobre todo cuando el lapso temporal disponible es corto. Otro factor que puede incidir negativamente en este tipo de situaciones es el tiempo de reacción que exhiben los niños más pequeños (menores de 9 años). Los tiempos de reacción en este grupo suelen ser más lentos que entre los niños mayores y pueden provocar la pérdida de oportunidades para cruzar o favorecer la realización de comportamientos riesgosos como correr durante el cruce.

Dado que muchas de las dificultades que enfrentan los niños cuando se movilizan como peatones se relacionan con sus capacidades psicomotrices, autores como Michon (1981), Van Schagen y Rothengatter (1997), o más recientemente Oxley y cols. (2008) han destacado la necesidad de que la educación vial incluya contenidos concretos sobre comportamientos seguros y entrenamiento de habilidades específicas en lugar de información abstracta sobre códigos y normas viales. El entrenamiento debe realizarse en contextos naturales con condiciones seguras o en escenarios virtuales, y debe incluir retroalimentación con respecto a las decisiones tomadas (Oxley, \& cols., 2008; Zeedick, \& cols., 2002).

Algo semejante ocurre cuando los niños y las niñas andan en bicicleta. La bicicleta es un medio de transporte que brinda independencia, permite recorrer grandes distancias y es divertido, sin embargo, conducir una bicicleta es una tarea compleja que requiere tanto de habilidades motrices como de capacidades cognitivas como la atención, el planeamiento o la toma de decisiones. Estas capacidades se desarrollan paulatinamente en los niños (Briem, \& Bengtsson, 2000; Briem, Radeborg, Salo, \& Bengtsson, 2004). En los menores de 12 años la habilidad para controlar la bicicleta o la aptitud para atender a los estímulos relevantes del ambiente aún no se han desarrollado totalmente (Briem \& cols., 2004). En consecuencia, cometen muchos errores que se ven reflejados en lesiones o incidente viales que involucran a otros vehículos.

Tal como sucede con las habilidades necesarias para cruzar una calle, el uso seguro de una bicicleta es algo que puede ser enseñado. De ese modo es posible reducir el número de errores y de lesiones sufridas. Una herramienta importante para eso es el desarrollo de programas de entrenamiento destinados a incrementar la capacidad de los niños para controlar la bicicleta (Ducheyne, DeBourdeaudhuij, Lenoir, \& Cardon, 2014; Ducheyne, DeBourdeaudhuij, Lenoir, Spittaels, \& Cardon, 2013). Entre estas capacidades se encuentran subir y bajar de la bicicleta, circular en línea recta, estimar distancias, señalar comportamientos, mirar por encima del hombro, o frenar.

También resulta relevante que durante la infancia se transmita que la bicicleta es un medio de transporte que tiene beneficios individuales, ambientales y sociales. Por ejemplo, 
a nivel de la salud individual, ir a la escuela en bicicleta ha sido asociado a la reducción del sedentarismo (Tudor-Locke, Neff, Ainsworth, Addy, \& Popkin, 2002) y a la disminución del índice de masa corporal y de la obesidad en niños (Pabayo, Gauvin, Barnett, Nikiema, \& Seguin, 2009). En términos ambientales, andar en bicicleta reduce la contaminación sonora y mejora la calidad del aire (Edward, \& Magarey, 2007). Según algunas estimaciones, si se reemplazara sólo el $1 \%$ de los viajes realizados en auto por viajes en bicicleta la contaminación ambiental se reduciría entre un 2 y un $4 \%$ (Gardner, 1998).

Viajar como pasajero en un transporte público o escolar, o en un vehículo familiar también implica riesgos y medidas específicas de prevención. Los niños deben aprender a desarrollar hábitos de auto-protección que incluyen cómo subir y bajar de los vehículos, en qué lugar ubicarse, y cómo usar correctamente los sistemas de sujeción. Cuando viajan como pasajeros los niños dependen en gran medida de las acciones que realizan los adultos. Por este motivo, unos y otros deben estar al tanto de la importancia de utilizar los mecanismos de seguridad existentes de manera adecuada. La evidencia indica que cuando los niños viajan en asientos traseros con dispositivos de sujeción específicos la probabilidad de resultar lesionados en un choque disminuye (Berg, Cook, Corneli, Vernon, \& Dean, 2000; Skjerven-Martinsen y cols. 2011).

En síntesis, la educación vial durante la infancia presenta el desafío de actuar con conocimiento sobre las limitaciones típicas de la edad y su interacción con un sistema vial que ha sido creado para otros usuarios. Frente a esta realidad, la tarea principal es proveer a los niños y niñas de conocimientos prácticos y dispositivos de seguridad adecuados. No alcanza con transmitir conceptualmente cuáles son las normas viales y la importancia de respetarlas. Además, resulta necesario favorecer el aprendizaje y el entrenamiento de estas habilidades en contextos seguros. Los adultos tienen un papel fundamental en la transmisión de esos conocimientos.

\section{Educación y Nuevas Necesidades en Materia de Transporte}

En la actualidad, el uso de vehículos personales de motor constituye la modalidad de transporte dominante. El uso de automóviles se ha masificado debido, entre otras razones, a los beneficios individuales de comodidad, velocidad e independencia en los desplazamientos (Gärling, \& Schuitema, 2007). No hay que olvidar además el valor simbólico del automóvil, que lo convierte en un objeto de consumo masivo (Steg, 2005). La expansión progresiva de esta forma hegemónica de movilidad, ha generado diversos problemas, como son la inseguridad de los usuarios más vulnerables, la pérdida de calidad ambiental, la inequidad en la distribución de oportunidades, la pérdida de cohesión social y el incremento del sedentarismo (Donaghy, Rudinger, \& Poppelreuter, 2004; Jacoby, \& Pardo, 2010; Tapia Granado, 1998).
Tal como fue mencionado anteriormente, un problema asociado al uso masivo de vehículos motorizados es la inseguridad vial que se traduce en un elevado número de muertes y lesiones alrededor del mundo. Esto ha dado lugar a que se considere a los siniestros viales como un problema de salud pública y se hayan generado numerosas recomendaciones para su tratamiento entre las que se destacan las realizadas desde la Organización Mundial de la Salud. Este organismo en su último informe (World Health Organization [WHO], 2013) señaló que la mitad de las muertes debidas al tránsito ocurren entre peatones, ciclistas y motociclistas. En consecuencia, sugiere que las políticas públicas deberían dirigirse a proteger a estos usuarios vulnerables mediante medidas específicas como la reducción de las velocidades máximas permitidas, o la generación de espacios exclusivos. Lamentablemente son pocos los países que han generado medidas concretas de este tipo hasta el momento.

Con respecto al sedentarismo existen estudios que plantean una relación positiva entre las tasas de obesidad y el uso del automóvil (Bassett, Pucher, Buehler, Thompson, \& Crouter, 2008; Jacobson, King, \& Yuan, 2011). Esto se observa claramente al comparar países de acuerdo con sus formas típicas de movilidad. Existen países, como es el caso de Suecia y Dinamarca, donde un porcentaje importante de los viajes urbanos se realiza en bicicleta o caminando, y los niveles de obesidad se mantienen bajos; a diferencia de otros como Brasil donde las tasas de obesidad han aumentado junto con el uso masivo del automóvil (Becerra, \& Scarlett, 2004).

Un aspecto asociado al sedentarismo entre los niños es la falta o el poco uso de medios activos de movilidad. En el caso de los niños son sus padres quienes suelen definir la manera en que se desplazarán de un lugar a otro. Un aspecto clave para que los padres permitan que sus hijos se desplacen de forma autónoma y activa es la percepción que tienen sobre la fortaleza de los lazos comunitarios (Waygood, \& Friman, 2015). Mientras más fuertes son esos lazos sociales mayor es la predisposición positiva de los padres para permitir el desplazamiento autónomo y activo de los niños. El uso intensivo del ambiente construido puede incrementar los lazos sociales siempre que se favorezca el uso de formas de movilidad alternativas al vehículo motorizado particular. Cuando eso no ocurre el nivel elevado de tránsito automotor afecta la interacción social, genera preocupación entre los padres por la seguridad de los niños y disminuye su predisposición a permitir que se desplacen de forma autónoma (Waygood \& Friman, 2015).

Pese al reconocimiento de los perjuicios mencionados, el contexto vial tiene características que favorecen el uso de vehículos personales motorizados en detrimento de formas de movilidad activas. Es el caso de la infraestructura de las calles, que generalmente se crea y modifica en favor de formas de movilidad a motor, sin tener en cuenta que el factor central debería ser la seguridad de los usuarios vulnerables. Del mismo modo, la educación vial infantil de manera indirecta facilita el uso de automóviles al pensar al niño como "pequeño conductor" o como "futuro conductor" repro- 
duciendo así la modalidad de transporte dominante. Para desarrollar iniciativas adecuadas es necesario considerar cuales son las formas típicas de desplazamiento durante la infancia. Los niños más que futuros conductores de automóviles son peatones, ciclistas o pasajeros. Muchas iniciativas se enfocan en una perspectiva a futuro y destacan aspectos relevantes para los conductores pero, en ocasiones, secundarios para los niños (por ejemplo, el aprendizaje de normas o comportamientos relacionados con la conducción). Al respecto, es ilustrativa una campaña realizada recientemente por la Agencia Nacional de Seguridad Vial (ANSV) y la empresa Yacimientos Petrolíferos Fiscales (YPF) denominada Ases al Volante. El objetivo de la campaña era que los niños tuvieran la experiencia de aprender activamente las normas de tránsito. Para ello se montaba una pista de karting que simulaba el contexto urbano con distintos paisajes de fondo. La pista incluía una estación de servicio completa, con todas sus instalaciones. Luego de recorrer el circuito todos los pequeños conductores recibían su "primera licencia de conducir".

De acuerdo con lo expuesto, parece claro que es necesario el desarrollo y la implementación de políticas enfocadas en la promoción y educación en el uso de medios de transporte alternativos al vehículo personal. Incentivar el uso del transporte público y de formas activas de movilidad (e. g. caminar, andar en bicicleta) deberían ser prioridades en los planes de desarrollo urbano. Sin embargo, según los datos ofrecidos por la OMS (WHO, 2013), son pocos los países que diseñan políticas que promuevan el uso de medios de transporte alternativos.

Algunos países podrían señalarse como ejemplo. Entre ellos se destacan Holanda o Alemania, que son pioneros en el desarrollo de intervenciones que favorecen e incentivan el transporte activo, y que han generado información científica al respecto (Pucher, \& Dijkstra, 2003). En el Reino Unido también se han llevado adelante investigaciones interesantes. Se ha estudiado sistemáticamente el transporte activo en niños, sus ventajas y desventajas y variables personales y contextuales asociadas. Entre otros resultados se observó que los niños que habitualmente caminan hacia la escuela son más activos que aquellos que se desplazan en auto (Mackett, \& Paskins, 2008). También se observó que la actitud de los niños hacia formas alternativas de movilidad se relaciona con las opiniones y actitudes parentales (e.g., Davidson, Davidson, Reed, Halden, \& Dillon, 2003). Específicamente, con la elección que los padres realizan sobre el medio de transporte en que sus hijos concurren a la escuela. Distintos autores señalaron que la elección del automóvil se ve favorecida por factores tanto contextuales como psicológicos (Davidson y cols., 2003; Lorenc, Brunton, Oliver, Oliver, \& Oakley, 2008). Entre los factores contextuales se encuentran las distancias a recorrer, y la evaluación del ambiente en términos de seguridad y preferencia. Entre las variables psicológicas, el valor simbólico del automóvil, o la necesidad de cumplir expectativas personales y sociales tienen un peso importante (Lorenc y cols. 2008).
En Latinoamérica es incipiente el interés en el transporte activo y sustentable. En consecuencia, el desarrollo de políticas públicas al respecto es escaso. Se observan excepciones aisladas a este patrón común. Algunos ejemplos son la ciudad de Curitiba en Brasil, Bogotá en Colombia, y recientemente, Buenos Aires, en Argentina. La sociedad civil se ha mostrado un poco más activa, y en distintos países existen organizaciones no gubernamentales con iniciativas muy valiosas. Por ejemplo, en Chile, la organización Ciudad Viva, incentiva la movilidad activa como medio para lograr una mayor equidad y sustentabilidad en el transporte y mejorar la calidad de los espacios públicos. Otras organizaciones con objetivos similares son Ciclópolis en Ecuador, Transporte Activo en Brasil y la Asociación de Ciclistas Urbanos en Argentina. Muchas de ellas se agrupan en una red mayor denominada Sustainable Transport Action Network-Latin America (SustranLac). El principal objetivo de esta red es la promoción del transporte activo como medio para resolver los problemas asociados a la inseguridad vial, la contaminación ambiental y la falta de acceso equitativo al transporte y otros servicios públicos.

En Argentina, la difusión de los conceptos de transporte activo, sustentable y equitativo es reciente. Su inclusión en los programas de educación vial permitiría aumentar el conocimiento de estos conceptos y desarrollar actitudes positivas hacia formas alternativas de transporte.

\section{¿Es Suficiente Educar?}

Es indudable que la educación tiene un papel relevante para mejorar las condiciones de seguridad vial. Sin embargo, sería erróneo creer que es el único factor o el más preponderante para lograr cambios de comportamiento. En psicología del tránsito se utiliza una expresión que resume los tres aspectos que componen las intervenciones necesarias y exitosas en términos de seguridad vial. Se habla de las Tres E (por sus siglas en inglés - education, enforcement and engineering) para hacer referencia a la educación, el control y la ingeniería (Groeger, 2011). Estos tres elementos son consonantes con otra tríada necesaria para entender los hechos que ocurren en el contexto del tránsito destacada por los epidemiólogos: el comportamiento humano, el vehículo y las condiciones ambientales, es decir, la vía y el medio socio-cultural.

Una primera aproximación llevaría a suponer que existe una relación uno a uno entre cada uno de estos factores. Así, la educación impactaría directamente sobre el comportamiento del conductor, la ingeniería actuaría sobre las condiciones de seguridad de la vía y del vehículo, y el control actuaría sobre la cultura vial (Warner, Ozkän, Lajunen, \& Tzamalouka, 2011). Sin embargo, las relaciones entre estos elementos son más complejas.

Estas relaciones pueden ilustrarse tanto para la seguridad vial como para la promoción de medios activos de movilidad. En cuanto a la seguridad vial, distintos autores han informado que la reducción en las tasas de siniestros 
y lesiones responden a campañas de intervención sistemáticas. Las campañas exitosas incluyen acciones multinivel que involucran cambios legislativos, aumento de los controles policiales, y campañas masivas de educación e información (OMS, 2009; Nazif, 2011).

El uso de formas activas de movilidad también se ve influido por diversos factores. Distintos estudios realizados en países de altos ingresos indicaron que variables como la densidad habitacional (cantidad de unidades habitacionales por hectárea) y la diversidad en el uso de los espacios construidos, son variables ambientales que incentivan la movilidad activa (Kyttä, Broberg, \& Kahila, 2012). En países con economías de menores ingresos per cápita, la elección de estas formas de movilidad parece responder a otras claves ambientales. Por ejemplo, en Colombia, en la ciudad de Bogotá, Cervero, Sarmiento, Jacoby, Gómez, y Neiman (2009) observaron que la densidad de las calles y la conectividad entre las vías diseñadas para andar en bicicleta o caminar se relacionan con la selección del medio de transporte. Estos investigadores no obtuvieron evidencia acerca de la influencia del espacio construido. En cuanto a las intervenciones destinadas a lograr cambios en el comportamiento, en el Reino Unido se realizaron una serie de medidas que incluyeron la modificación del espacio vial mediante la construcción de ciclovías, la pacificación del tránsito (traffic calming), la disminución de los límites de velocidad y hasta un impuesto a la congestión vehicular. Todas estas medidas produjeron en el año 2010 la incorporación de más de un millón de nuevos ciclistas en ese conjunto de países (Grous, 2011).

En síntesis, los cambios en el comportamiento vial son consecuencia de acciones realizadas en varios niveles. La educación es una acción importante pero no es el único elemento en el que se deben apoyar los programas e intervenciones. La educación apela a los individuos. De ellos se espera que se comporten más racionalmente o de acuerdo con el mejor conocimiento adquirido. Sin embargo, si las variables contextuales no facilitan o apoyan esos cambios la educación puede convertirse en una actividad en el vacío. Por ese motivo, son fundamentales las intervenciones a nivel ambiental, ya sea en el diseño de espacios urbanos o en términos de legislación y control. Este tipo de intervenciones no son una responsabilidad individual sino que recaen sobre quienes toman decisiones y establecen políticas públicas.

\section{Conclusiones}

La educación vial durante la infancia es un factor relevante para prevenir los riesgos que se enfrentan en el contexto del tránsito. Sin embargo, las modificaciones experimentadas por la creciente motorización de los desplazamientos generan la necesidad de un cambio de enfoque. Por un lado, resulta importante desarrollar perspectivas que destaquen las necesidades específicas de la niñez en el tránsito. Por otro, es importante que se trabaje en la generación de condiciones ambientales más amigables, seguras y sostenibles.
Si uno de los objetivos de la educación es la prevención de siniestros y sus consecuencias, también es importante poder conocer las condiciones, factores de riesgo y mecanismos de lesión que afectan a los más chicos. Por ello, es importante destacar la necesidad de investigaciones sobre factores de riesgo específicos. Resulta imprescindible tener en cuenta que el diseño de intervenciones no puede realizarse sin conocimiento previo de las características del grupo o destinatario con el cual se va a realizar una intervención. En este sentido y en el caso particular de la infancia, es claro que se requieren intervenciones adecuadas a las características y riesgos típicos de este grupo.

También resulta necesario ir más allá de la concepción tradicional de educación vial infantil. El enfoque del 'pequeño conductor' debería ser reemplazado por una perspectiva dónde se contemplen las formas de desplazamiento características de las distintas etapas evolutivas de la infancia y los factores de riesgo a ellas asociadas. Los datos epidemiológicos indican que para este grupo de edad los siniestros más comunes ocurren como peatones, ciclistas o pasajeros. Por lo tanto, no parece del todo adecuado trabajar solamente sobre el aprendizaje de normas y señales viales. Además, vale preguntarse si el aprendizaje de normas que no son respetadas no supone un riesgo añadido. Al respecto, resulta imprescindible que los adultos modifiquen sus comportamientos ya que independientemente del riesgo que generan la contravención de las normas, las acciones de los adultos actúan modelando el comportamiento futuro de los niños.

Uno de los desafíos de la educación vial es promover comportamientos seguros en el tránsito, pero sin restringir el derecho a los desplazamientos y la movilidad. No debería olvidarse que la exploración del espacio constituye una actividad necesaria para el desarrollo, y la construcción de lazos sociales. Como parte del desarrollo normal de la infancia, los niños y niñas deberían poder explorar, conocer y disfrutar de su entorno barrial. Los riesgos del tránsito son una barrera para estas posibilidades de exploración. Por ello, una parte importante del trabajo debería consistir en facilitar sus desplazamientos garantizando la seguridad del entorno, lo cual básicamente es una tarea del Estado. Las políticas públicas deberían ocuparse de la modificación de los ambientes viales para favorecer su uso multimodal. Al Estado y a los gobernantes les corresponde también ejercer el control necesario para que las normas sean respetadas.

Una visión de la educación vial centrada solamente en la seguridad deja a un lado aspectos relevantes para el transporte en contextos urbanos. La accesibilidad y la sustentabilidad del transporte son dimensiones de gran relevancia. Frente a la creciente motorización de los desplazamientos la educación tendría que incentivar una perspectiva crítica sobre el transporte y las soluciones posibles en un futuro cercano. Entre los problemas que requieren ser discutidos se encuentran las consecuencias del uso generalizado de vehículos personales motorizados, y los beneficios individuales y colectivos de las modalidades públicas y activas de transporte. En síntesis, la educación vial infantil debe 
avanzar hacia la formación en valores para un tránsito más seguro, responsable, saludable y equitativo.

\section{Referencias}

Agencia Nacional de Seguridad Vial [ANSV]. (2011). Programa de Fortalecimiento de la Educación Vial. Recuperado: 20 mar. 2014. Disponível: http://www.educacionvial.gov.ar.

Bassett, D. R., Pucher, J., Buehler, R., Thompson, D. L., \& Crouter, S. E. (2008). Walking, cycling and obesity rates in Europe, North America and Australia. Journal of Physical Activity and Health, 5, 795-814.

Becerra, B., \& Scarlett, T. (2004). La caminabilidad de las ciudades como un reflejo del desarrollo sustentable. Avances en energías Renovables y Medio Ambiente, 8, 94-98.

Berg, M. D., Cook, L., Corneli, H. M., Vernon, D. D., \& Dean, J. M. (2000). Effect of Seating Position and Restraint Use on Injuries to Children in Motor Vehicle Crashes. Pediatrics, 105, 831-835.

Briem, V., \& Bengtsson, H. (2000). Cognition and character traits as determinants of young children's behaviour in traffic situations. International Journal of Behavioral Development, 24, 492-505.

Briem, V., Radeborg, K., Salo, I., \& Bengtsson, H. (2004). Developmental aspects of children behavior and safety while cycling. Journal of Pediatrics Psychology, 29, 369-377.

Cervero, R., Sarmiento, O. L., Jacoby, E., Gomez, L. F., \& Neiman, A. (2009). Influences of built environments on walking and cycling: lessons from Bogotá. International Journal of Sustainable Transportation, 3, 203-226.

Davidson, P., Davidson, P., Reed, N., Halden, D., \& Dillon, J. (2003). Children 's Attitudes to Sustainable Transport. Scottish Executive Social Research, 174, 1-4.

Donaghy, K., Rudinger, G., \& Poppelreuter, S. (2004). Societal trends, mobility behaviour and sustainable transport in Europe and North America. Transport Reviews, 24, 679-690.

Ducheyne, F., DeBourdeaudhuij, I., Lenoir, M., \& Cardon, G. (2014). Effects of a cycle training course on children's cycling skills and levels of cycling to school. Accident Analysis and Prevention, 67, 49-60.

Ducheyne, F., DeBourdeaudhuij, I., Lenoir, M., Spittaels, H., \& Cardon, G. (2013). Children cycling skills: Development of a test and determination of individual and environmental correlates. Accident Analysis \& Prevention, 50, 688-697.

Durkin, M. S., Laraque, D., Lubman, I., \& Barlow, B. (1999). Epidemiology and prevention of traffic injuries to urban children and adolescents. Pediatrics, 6, 74-82.
Edward, S., \& Magarey, P. (2007). Environmental benefits of cycling. Cycling Promotion Fund and Bicycle Federation of Australia. Recuperado: 21 fev. 2014. Disponível: http://www. cycling romotion.com.au/images/stories/factsheets/02 TheEnvironmentalBenefitsOfCycling.pdf

Fleitas, D. (2010). Accidentes de tránsito en la Argentina. Recuperado: 10 abr. 2014. Disponível: http://www.app.org.ar/wp-content/ uploads/2011/04/Accidentes-de-Transito-en-Argentina-2010-final. pdf

Gardner, G. (1998). When cities take bicycles seriously. World Watch. Disponível: http://infohouse.p2ric.org/ref/37/36221.pdf

Gärling, T., \& Schuitema, G (2007). Travel Demand Management Targeting Reduced Private Car Use: Effectiveness, Public Acceptability and Political Feasibility. Journal of Social Issues, 63 , 139-153.

Groeger, J. A (2011). How many E's in Road Safety. Em B. E. Porter (Org.), Handbook of traffic psychology (pp.3-70). United States of America: Academic Press, Elsevier.

Grous, A. (2011). The British Cycling Economy. 'Gross cycling Product' Report. London School of Economics. Recuperado: 20 mar. 2014. Disponível: http://corporate.sky.com/documents/pdf/publications/ the_british_cycling_economy

Jacobson, S. H., King, D. M., K., \& Yuan, R. (2011). A note on the relationship between obesity and driving. Transport Policy, 18, 772 776 .

Jacoby, E., \& Pardo, C. F (2010). Ciudades del automóvil, obesidad y cambio climático: se alinean las crisis y también las soluciones. Serie Discusiones Contemporáneas. Sustran Lac. Recuperado: mar. 2014. Disponível: http://www.bvsde.paho.org /texcom/ cd045364/ciudadesauto.pdf

Kyttä, M. A., Broberg, A. K., \& Kahila, M. H. (2012). Urban Environment and Children's Active Lifestyle: Soft GIS Revealing Children's Behavioral Patterns and Meaningful Places. American Journal of Health Promotion, 26, 137-148.

Lorenc, T., Brunton, G., Oliver, S., Oliver, K., \& Oakley, A. (2008). Attitudes to walking and cycling among children young people and parents: a systematic review. Journal of Epidemiology and Community Health, 62, 852-857.

National Safe Kids Campaign. (2004). Bicycle Injury Fact Sheet the National SAFE KIDS campaign. Washington, DC.

Nazif, J. I. (2011). Guía práctica para el diseño e implementación de políticas de seguridad vial integrales considerando el rol de la infraestructura. Naciones Unidas, Comisión Económica para América Latina y el Caribe. Disponível: http://www.eclac.org/ publicaciones/xml/8/43158/Lcw380el.pdf 
Michon, (1981). Traffic Education for young pedestrians: An introduction. Accident Analysis and Prevention, 13, 163-167.

Mackett, R., \& Paskins, J (2008). Children's Physical Activity: The Contribution of Playing and Walking. Children \& Society, 22, 345357.

Organización Mundial de la Salud [OMS]. (2008). Informe mundial sobre prevención de lesiones en los niños. Disponível: http://apps. who.int/iris/bitstream /10665/77762/1/WHO_NMH_VIP08.01_spa. pdf

Organización Mundial de la Salud [OMS]. (2009). Informe sobre la situación mundial de la seguridad vial: es hora de pasar a la acción. Recuperado: 21 jan. 2014. Disponível: http://www.who.int/ violence_injury_prevention/road_safety_status/report/cover_and_ front_matter_es.pdf

Oxley, J., Congiu, M., Whelan, M., D’Elio, A., \& Charlton, J. (2008). Teaching young children to cross roads safely. Annals of Advancement of Automotive Medicine, 52, 215-226.

Pabayo, R., Gauvin, L., Barnett, T. A., Nikiema, B., \& Seguin, L. (2009). Sustained Active Transportation is associated with a favorable body mass index trajectory across the early school years: Findings from the Quebec Longitudinal Study of Child Development birth cohort. Preventive Medicine, 6, 59-64.

Pucher, J., \& Dijkstra, L. (2003). Promoting safe walking and cycling to improve public health: Lessons from the Netherlands and Germany. American Journal of Public Health, 9, 1509-1516.

Romano, E,. \& Kelley-Baker, T. (2015). Child passenger injured in motor vehicle crashes. Journal of Safety Research, 52, 1-8.

Skjerven-Martinsen, M., Naess, P.A., Hansen, T. B., Rognum, T. O., Lereim, I., \& Stray-Pedersen, A. (2011). In-depth evaluation of real-world car collisions: fatal and severe injuries in children are predominantly caused by restraint errors and unstrapped cargo. Traffic Injury Prevention, 12, 491-499.
Steg, L. (2005). Car use: Lust and must. Instrumental, symbolic and affective motives for car use. Transportation Research Part A: Policy and Practice, 39, 147-162.

Tapia Granado, J. A. (1998). La reducción del tráfico de automóviles: una política urgente de promoción de la salud. Revista Panamericana de Salud Pública, 3, 137-151.

Tudor-Locke, C., Neff, L. J., Ainsworth, B. E., Addy, C. L., \& Popkin, B.M. (2002). Omissions of active commuting to school and the prevalence of children health-related physical activity levels: The Russian Longitudinal Monitoring Study. Child: Care, Health and Development, 28, 507-512.

Van Schagen, I., \& Rothengatter, T. (1997). Classroom instruction vs roadside training in traffic safety education. Journal of Applied Developmental Psychology, 18, 283-292.

Waisman, I., Nuñez, J. M., \& Sánchez, J. (2002). Epidemiología de los accidentes en la infancia en la Región Centro de Cuyo. Revista Chilena de Pediatría, 73, 404-414.

Warner, H. W., Özkan, T., Lajunen, T., \& Tzamalouka, G. (2011). Cross-cultural comparison of drivers' tendency to commit different aberrant driving behaviours. Transportation Research Part F: Traffic Psychology and Behaviour, 14, 390-399.

Waygood, E. O. D., \& Friman, M. (2015). Children's travel and incidental community connections. Travel Behaviour and Society, 2, 174-181.

Wolff, S. P., \& Gillham, C. J. (1991). Public health versus Public policy? An appraisal of British urban transport policy. Public Health, 105, 217-228.

World Health Organization [WHO]. (2013). Global status report on road Safety. World Health Organization. Geneva, Switzerland.

Zeedick, M. S., Wallace, L., \& Spry, L. (2002). Stop, look, listen, and think? Accident Analysis and Prevention, 34, 43-50. 


\section{Sobre os autores}

Fernando Martín Poó (poo.fernando@gmail.com)

Dr. en Psicología. Investigador Asistente del Consejo Nacional de Investigaciones Científicas y Técnicas (CONICET). Docente en la Facultad de Psicología de la Universidad Nacional de Mar del Plata. Miembro del Grupo de Investigación Modelos y Métodos de Investigación en Psicología aplicada al Tránsito.

Soledad Susana López (soledad.lopez@conicet.gov.ar)

Licenciada en Psicología, estudiante de doctorado y becaria de postgrado del Consejo Nacional de Investigaciones Científicas y Técnicas (CONICET). Miembro del Grupo de Investigación Modelos y Métodos de Investigación en Psicología aplicada al Tránsito.

Jeremías Tosi (jeremiastosi@gmail.com)

Licenciado en Psicología. Becario de la Universidad Nacional de Mar del Plata. Docente de la Facultad de Psicología de la Universidad Nacional de Mar del Plata. Miembro del Grupo de Investigación Modelos y Métodos de Investigación en Psicología aplicada al Tránsito.

María Isabel Nucciarone (mariaisabelnucciarone@gmail.com)

Licenciada en Psicología. Miembro del Grupo de Investigación Modelos y Métodos de Investigación en Psicología aplicada al Tránsito.

Rubén Daniel Ledesma (rdledesma@gmail.com)

Dr. en Psicología. Investigador Independiente del Consejo Nacional de Investigaciones Científicas y Técnicas (CONICET). Docente en la Facultad de Ciencias de la Salud de la Universidad Nacional de Mar del Plata. Director del Grupo de Investigación Modelos y Métodos de Investigación en Psicología aplicada al Tránsito

El presente trabajo cuenta con la financiación de CONICET y la UNMdP. Una versión preliminar fue presentada en el Congreso Iberoamericano de Educación, Metas 2021 realizado en el año 2010. La versión actual fue escrita durante los meses de febrero y abril del año 2013. 
